\title{
ESTIMATES FOR SINGULAR INTEGRALS ALONG SURFACES OF REVOLUTION
}

\author{
SHUICHI SATO
}

(Received 27 November 2006; accepted 8 May 2008)

Communicated by G. A. Willis

\begin{abstract}
We prove certain $L^{p}$ estimates $(1<p<\infty)$ for nonisotropic singular integrals along surfaces of revolution. The singular integrals are defined by rough kernels. As an application we obtain $L^{p}$ boundedness of the singular integrals under a sharp size condition on their kernels. We also prove a certain estimate for a trigonometric integral, which is useful in studying nonisotropic singular integrals.
\end{abstract}

2000 Mathematics subject classification: primary 42B20, 42B25.

Keywords and phrases: nonisotropic singular integrals, extrapolation, trigonometric integrals.

\section{Introduction}

Let $P$ be an $n \times n$ real matrix whose eigenvalues have positive real parts. Let $\gamma=\operatorname{trace} P$. Define a dilation group $\left\{A_{t}\right\}_{t>0}$ on $\mathbb{R}^{n}$ by $A_{t}=t^{P}=\exp ((\log t) P)$. We assume that $n \geq 2$. There is a nonnegative function $r$ on $\mathbb{R}^{n}$ associated with $\left\{A_{t}\right\}_{t>0}$. The function $r$ is continuous on $\mathbb{R}^{n}$ and infinitely differentiable in $\mathbb{R}^{n} \backslash\{0\}$. Furthermore, it satisfies the following conditions.

(1) $\quad r\left(A_{t} x\right)=\operatorname{tr}(x)$ for all $t>0$ and $x \in \mathbb{R}^{n}$.

(2) $r(x+y) \leq C(r(x)+r(y))$ for some $C>0$.

(3) If $\Sigma=\left\{x \in \mathbb{R}^{n} \mid r(x)=1\right\}$, then $\Sigma=\left\{\theta \in \mathbb{R}^{n} \mid\langle B \theta, \theta\rangle=1\right\}$ for a positive symmetric matrix $B$, where $\langle\cdot, \cdot\rangle$ denotes the inner product in $\mathbb{R}^{n}$.

Also $d x=t^{\gamma-1} d \sigma d t$, that is,

$$
\int_{\mathbb{R}^{n}} f(x) d x=\int_{0}^{\infty} \int_{\Sigma} f\left(A_{t} \theta\right) t^{\gamma-1} d \sigma(\theta) d t
$$

for appropriate functions $f$, where $d \sigma$ is a $C^{\infty}$ measure on $\Sigma$. See $[2,13,17]$ for more details.

(C) 2009 Australian Mathematical Society 1446-7887/2009 \$16.00 
Let $\Omega$ be locally integrable in $\mathbb{R}^{n} \backslash\{0\}$ and homogeneous of degree 0 with respect to the dilation group $\left\{A_{t}\right\}$, that is, $\Omega\left(A_{t} x\right)=\Omega(x)$ for $x \neq 0$. We assume that

$$
\int_{\Sigma} \Omega(\theta) d \sigma(\theta)=0
$$

For $s \geq 1$, let $\Delta_{s}$ denote the collection of measurable functions $h$ on $\mathbb{R}_{+}=\{t \in \mathbb{R} \mid t>$ 0\} satisfying

$$
\|h\|_{\Delta_{s}}=\sup _{j \in \mathbb{Z}}\left(\int_{2^{j}}^{2^{j+1}}|h(t)|^{s} d t / t\right)^{1 / s}<\infty,
$$

where $\mathbb{Z}$ denotes the set of integers. We define $\|h\|_{\Delta_{\infty}}$ as usual $\left(\|h\|_{\Delta_{\infty}}=\|h\|_{L^{\infty}\left(\mathbb{R}_{+}\right)}\right)$.

Let $\Gamma:[0, \infty) \rightarrow \mathbb{R}^{m}$ be a continuous mapping satisfying $\Gamma(0)=0$. We define a singular integral operator along the surface $(y, \Gamma(r(y)))$ by

$$
\begin{aligned}
T f(x, z) & =\text { p.v. } \int_{\mathbb{R}^{n}} f(x-y, z-\Gamma(r(y))) K(y) d y \\
& =\lim _{\epsilon \rightarrow 0} \int_{r(y)>\epsilon} f(x-y, z-\Gamma(r(y))) K(y) d y,
\end{aligned}
$$

where $K(y)=h(r(y)) \Omega\left(y^{\prime}\right) r(y)^{-\gamma}, y^{\prime}=A_{r(y)^{-1}} y$ and $h \in \Delta_{1}$. We assume that the principal value integral in (1.1) exists for all $(x, z) \in \mathbb{R}^{n} \times \mathbb{R}^{m}$ and $f \in \mathcal{S}\left(\mathbb{R}^{n} \times \mathbb{R}^{m}\right)$ (the Schwartz class).

We denote by $L \log L(\Sigma)$ the Zygmund class of all those functions $\Omega$ on $\Sigma$ which satisfy

$$
\int_{\Sigma}|\Omega(\theta)| \log (2+|\Omega(\theta)|) d \sigma(\theta)<\infty .
$$

Also, we consider the $L^{q}(\Sigma)$ spaces and write $\|\Omega\|_{q}=\left(\int_{\Sigma}|\Omega(\theta)|^{q} d \sigma(\theta)\right)^{1 / q}$ for $\Omega \in L^{q}(\Sigma)\left(\|\Omega\|_{\infty}\right.$ is defined as usual).

Let

$$
M_{\Gamma} g(z)=\sup _{R>0} R^{-1} \int_{0}^{R}|g(z-\Gamma(t))| d t .
$$

We assume that the maximal operator $M_{\Gamma}$ is bounded on $L^{p}\left(\mathbb{R}^{m}\right)$ for all $p>1$. See $[15,17]$ for examples of such functions $\Gamma$.

In this note we prove the following theorems.

TheOREM 1.1. Let $T$ be as in (1.1). Suppose that $\Omega \in L^{q}(\Sigma)$ for some $q \in(1,2]$ and $h \in \Delta_{s}$ for some $s>1$. Then

$$
\|T f\|_{L^{p}\left(\mathbb{R}^{n+m}\right)} \leq C_{p}(q-1)^{-1}\|\Omega\|_{q}\|h\|_{\Delta_{s}}\|f\|_{L^{p}\left(\mathbb{R}^{n+m}\right)}
$$

if $|1 / p-1 / 2|<\min \left(1 / s^{\prime}, 1 / 2\right)$, where $1 / s^{\prime}+1 / s=1$ and the constant $C_{p}$ is independent of $q$ and $\Omega$. 
THEOREM 1.2. Suppose that $\Omega \in L \log L(\Sigma)$ and $h \in \Delta_{s}$ for some $s>1$. Then $T$ is bounded on $L^{p}\left(\mathbb{R}^{n+m}\right)$ if $|1 / p-1 / 2|<\min \left(1 / s^{\prime}, 1 / 2\right)$.

Theorem 1.2 follows from Theorem 1.1 by an extrapolation method. When $r(x)=|x|$ (the Euclidean norm), $m=1$ and $\Gamma$ is a $C^{2}$, convex, increasing function, Theorem 1.2 was proved in Al-Salman and Pan [1] (see [1, Theorem 4.1] and also [10] for a related result). In [1], it is noted that the estimates as $q \rightarrow 1$ of Theorem 1.1 (in their setting) can be used through extrapolation to prove the $L^{p}$ boundedness of [1, Theorem 4.1], although such estimates are yet to be proved. In this note, we are able to prove Theorem 1.1 and apply it to prove Theorem 1.2.

If $\Gamma \equiv 0$ ( $\Gamma$ is identically 0$)$, then $T$ essentially reduces to the lower-dimensional singular integral

$$
S f(x)=\text { p.v. } \int_{\mathbb{R}^{n}} f(x-y) K(y) d y .
$$

For this singular integral we have the following theorem.

THEOREM 1.3. Let $\Omega \in L^{q}(\Sigma)$ and $h \in \Delta_{s}$ for some $q, s \in(1,2]$. Then

$$
\|S f\|_{L^{p}\left(\mathbb{R}^{n}\right)} \leq C_{p}(q-1)^{-1}(s-1)^{-1}\|\Omega\|_{q}\|h\|_{\Delta_{s}}\|f\|_{L^{p}\left(\mathbb{R}^{n}\right)}
$$

for all $p \in(1, \infty)$, where the constant $C_{p}$ is independent of $q, s, \Omega$ and $h$.

For $a>0$, let

$$
L_{a}(h)=\sup _{j \in \mathbb{Z}} \int_{2^{j}}^{2^{j+1}}|h(r)|(\log (2+|h(r)|))^{a} d r / r .
$$

We define a class $\mathcal{L}_{a}$ to be the space of all those measurable functions $h$ on $\mathbb{R}_{+}$which satisfy $L_{a}(h)<\infty$.

By Theorem 1.3 and an extrapolation we have the following result.

THEOREM 1.4. Suppose that $\Omega \in L \log L(\Sigma)$ and $h \in \mathcal{L}_{a}$ for some $a>2$. Then $S$ is bounded on $L^{p}\left(\mathbb{R}^{n}\right)$ for all $p \in(1, \infty)$.

It is noted in [5] that $S$ is bounded on $L^{p}, 1<p<\infty$, if $\Omega \in L^{q}$ for some $q>1$ and $h \in \Delta_{2}$ (see [5, Corollary 4.5]). Theorem 1.4 improves that result. See [13, 16] for nonisotropic singular integrals $S$ with $h \equiv 1$ and also [3, 7, 9, 12] for related results.

In Section 2, we prove Theorems 1.1 and 1.3. The proofs are based on the method of [5]. As in [14], a key idea of the proof of Theorem 1.1 is to use a LittlewoodPaley decomposition depending on $q$ for which $\Omega \in L^{q}$. Theorem 1.3 is proved in a similar fashion. Applying an extrapolation argument, we can prove Theorems 1.2 and 1.4 from Theorems 1.1 and 1.3, respectively. We give a proof of Theorem 1.4 in Section 3. In Section 4, we prove an estimate for a trigonometric integral, a corollary of which is used in proving Theorems 1.1 and 1.3.

Throughout this note, the letter $C$ will be used to denote nonnegative constants which may be different in different occurrences. 


\section{Proofs of Theorems 1.1 and 1.3}

Let $A^{*}$ denote the adjoint of a matrix $A$. Then $A_{t}^{*}=\exp \left((\log t) P^{*}\right)$. We write $A_{t}^{*}=B_{t}$. We can define a nonnegative function $s$ from $\left\{B_{t}\right\}$ in exactly the same way as we define $r$ from $\left\{A_{t}\right\}$.

There are positive constants $c_{1}, c_{2}, c_{3}, c_{4}, \alpha_{1}, \alpha_{2}, \beta_{1}$ and $\beta_{2}$ such that

$$
\begin{array}{ll}
c_{1}|x|^{\alpha_{1}}<r(x)<c_{2}|x|^{\alpha_{2}} & \text { if } r(x) \geq 1, \\
c_{3}|x|^{\beta_{1}}<r(x)<c_{4}|x|^{\beta_{2}} & \text { if } 0<r(x) \leq 1 .
\end{array}
$$

Also,

$$
\begin{array}{ll}
d_{1}|\xi|^{a_{1}}<s(\xi)<d_{2}|\xi|^{a_{2}} & \text { if } s(\xi) \geq 1, \\
d_{3}|\xi|^{b_{1}}<s(\xi)<d_{4}|\xi|^{b_{2}} & \text { if } 0<s(\xi) \leq 1,
\end{array}
$$

for some positive numbers $d_{1}, d_{2}, d_{3}, d_{4}, a_{1}, a_{2}, b_{1}$ and $b_{2}$ (see [17]). These estimates are useful in the following.

We consider the singular integral operator $T$ defined in (1.1). Let $E_{j}=\left\{x \in \mathbb{R}^{n} \mid\right.$ $\left.\beta^{j}<r(x) \leq \beta^{j+1}\right\}$, where $\beta \geq 2$ and $j \in \mathbb{Z}$. We define a sequence of Borel measures $\left\{\sigma_{j}\right\}$ on $\mathbb{R}^{n} \times \mathbb{R}^{m}$ by

$$
\hat{\sigma}_{j}(\xi, \eta)=\int_{E_{j}} \exp (-2 \pi i\langle y, \xi\rangle) \exp (-2 \pi i\langle\Gamma(r(y)), \eta\rangle) K(y) d y,
$$

where $\hat{\sigma}_{j}$ denotes the Fourier transform of $\sigma_{j}$ defined by

$$
\hat{\sigma}_{j}(\xi, \eta)=\int \exp (-2 \pi i\langle(x, z),(\xi, \eta)\rangle) d \sigma_{j}(x, z) .
$$

Then $T f(x)=\sum_{-\infty}^{\infty} \sigma_{k} * f(x)$.

Let $\mu_{k}=\left|\sigma_{k}\right|$, where $\left|\sigma_{k}\right|$ denotes the total variation of $\sigma_{k}$. Let $\Omega \in L^{q}, h \in \Delta_{s}$, $q, s \in(1,2]$. We prove the following estimates:

$$
\left\|\sigma_{k}\right\| \leq C(\log \beta)\|\Omega\|_{1}\|h\|_{\Delta_{1}} \leq C(\log \beta)\|\Omega\|_{q}\|h\|_{\Delta_{s}},
$$

where $\left\|\sigma_{k}\right\|=\left|\sigma_{k}\right|\left(\mathbb{R}^{n+m}\right)$;

$$
\left|\hat{\sigma}_{k}(\xi, \eta)\right| \leq C\|\Omega\|_{q}\|h\|_{\Delta_{s}}\left(\beta^{k+d} s(\xi)\right)^{1 / b_{1}}
$$

where $d=b_{1} / \alpha_{1}$;

$$
\left|\hat{\sigma}_{k}(\xi, \eta)\right| \leq C(\log \beta)\|\Omega\|_{q}\|h\|_{\Delta_{s}}\left(\beta^{k} s(\xi)\right)^{-\epsilon_{0} /\left(q^{\prime} s^{\prime}\right)}
$$

for some $\epsilon_{0}>0$;

$$
\left|\hat{\mu}_{k}(\xi, \eta)\right| \leq C(\log \beta)\|\Omega\|_{q}\|h\|_{\Delta_{s}}\left(\beta^{k} s(\xi)\right)^{-\epsilon_{0} /\left(q^{\prime} s^{\prime}\right)},
$$


where $\epsilon_{0}$ is as in (2.3); and

$$
\left|\hat{\mu}_{k}(\xi, \eta)-\hat{\mu}_{k}(0, \eta)\right| \leq C\|\Omega\|_{q}\|h\|_{\Delta_{s}}\left(\beta^{k+d} s(\xi)\right)^{1 / b_{1}},
$$

where $d$ is as in (2.2).

First, we see that

$$
\left\|\sigma_{k}\right\|_{1}=\int_{\beta^{k}}^{\beta^{k+1}}|h(r)|\|\Omega\|_{1} d r / r \leq C(\log \beta)\|\Omega\|_{1}\|h\|_{\Delta_{1}} .
$$

From this, (2.1) follows.

Next, we show (2.2). Take $v \in \mathbb{Z}$ so that $2^{v}<\beta \leq 2^{v+1}$. Note that

$$
\begin{aligned}
\hat{\sigma}_{k}(\xi, \eta)= & \int_{\beta^{k}<r(x) \leq \beta^{k+1}} \exp (-2 \pi i\langle\Gamma(r(x)), \eta\rangle)(\exp (-2 \pi i\langle x, \xi\rangle)-1) h(r(x)) \\
& \times \Omega\left(x^{\prime}\right) r(x)^{-\gamma} d x .
\end{aligned}
$$

Thus

$$
\begin{aligned}
\left|\hat{\sigma}_{k}(\xi, \eta)\right| & \leq C \int_{1<r(x) \leq \beta}|x|\left|B_{\beta^{k}} \xi\right|\left|h\left(\beta^{k} r(x)\right) \Omega\left(x^{\prime}\right)\right| r(x)^{-\gamma} d x \\
& \leq C \sum_{j=0}^{v}\left|B_{\beta^{k}} \xi\right|\|\Omega\|_{1} 2^{j / \alpha_{1}} \int_{2^{j}}^{2^{j+1}}\left|h\left(\beta^{k} r\right)\right| d r / r \\
& \leq C \beta^{1 / \alpha_{1}}\left|B_{\beta^{k}} \xi\right|\|\Omega\|_{1}\|h\|_{\Delta_{1}} .
\end{aligned}
$$

Combining (2.6) and (2.7),

$$
\left|\hat{\sigma}_{k}(\xi, \eta)\right| \leq C\|\Omega\|_{1}\|h\|_{\Delta_{1}} \min \left(\log \beta, \beta^{1 / \alpha_{1}}\left|B_{\beta^{k}} \xi\right|\right) .
$$

If $s\left(B_{\beta^{k}} \xi\right)<1$, then $\left|B_{\beta^{k}} \xi\right| \leq C\left(\beta^{k} s(\xi)\right)^{1 / b_{1}}$. Therefore

$$
\min \left(\log \beta, \beta^{1 / \alpha_{1}}\left|B_{\beta^{k}} \xi\right|\right) \leq C\left(\beta^{k+d} s(\xi)\right)^{1 / b_{1}} .
$$

Using this in (2.8), we have (2.2). We can prove (2.5) in the same way.

Next we prove (2.3). We use a method similar to that of [5, p. 551]. Define

$$
\tau(\xi)=\int_{\Sigma} \Omega(\theta) \exp (-2 \pi i\langle\xi, \theta\rangle) d \sigma(\theta) .
$$

We need the following estimates.

Lemma 2.1. Let $L$ be the degree of the minimal polynomial of $P$. Then, if $0<\epsilon_{0}$ $<a_{2}^{-1} \min \left(1 / 2, q^{\prime} / L\right)$,

$$
\int_{\beta^{k}}^{\beta^{k+1}}\left|\tau\left(B_{r} \xi\right)\right|^{2} d r / r \leq C(\log \beta)\left(\beta^{k} s(\xi)\right)^{-\epsilon_{0} / q^{\prime}}\|\Omega\|_{q}^{2},
$$

where $C$ is independent of $\Omega \in L^{q}, q \in(1,2]$ and $\beta$. 
In proving Lemma 2.1 we use the following estimate, which follows from the corollary to Theorem 4.1 in Section 4 via an integration by parts argument.

LEMMA 2.2. Let L be as in Lemma 2.1. Then, for $\eta, \zeta \in \mathbb{R}^{n} \backslash\{0\}$,

$$
\left|\int_{1}^{2} \exp \left(i\left\langle B_{t} \eta, \zeta\right\rangle\right) d t / t\right| \leq C|\langle\eta, P \zeta\rangle|^{-1 / L}
$$

for some positive constant $C$ independent of $\eta$ and $\zeta$.

Proof of Lemma 2.1. Choose $v \in \mathbb{Z}$ such that $2^{v}<\beta \leq 2^{v+1}$. Then

$$
\begin{aligned}
& \int_{\beta^{k}}^{\beta^{k+1}}\left|\tau\left(B_{r} \xi\right)\right|^{2} d r / r \leq \sum_{j=0}^{\nu} \int_{\beta^{k} 2^{j}}^{\beta^{k} 2^{j+1}}\left|\tau\left(B_{r} \xi\right)\right|^{2} d r / r \\
& \quad=\sum_{j=0}^{\nu} \iint_{\Sigma \times \Sigma}\left(\int_{1}^{2} \exp \left(-2 \pi i\left\langle B_{\beta^{k} 2_{r} j} \xi, \theta-\omega\right\rangle\right) d r / r\right) \Omega(\theta) \bar{\Omega}(\omega) d \sigma(\theta) d \sigma(\omega) .
\end{aligned}
$$

By Lemma 2.2,

$$
\left|\int_{1}^{2} \exp \left(-2 \pi i\left\langle B_{\beta^{k} 2^{j} r} \xi, \theta-\omega\right\rangle\right) d r / r\right| \leq C\left|\left\langle B_{\beta^{k} 2^{j}} \xi, P(\theta-\omega)\right\rangle\right|^{-\epsilon},
$$

where $0<\epsilon \leq 1 / L$. Using Hölder's inequality, if $0<\epsilon<\min \left(1 /\left(2 q^{\prime}\right), 1 / L\right)$, then

$$
\begin{aligned}
& \iint_{\Sigma \times \Sigma}\left|\left\langle B_{\beta^{k} 2^{j}} \xi, P(\theta-\omega)\right\rangle\right|^{-\epsilon}|\Omega(\theta) \bar{\Omega}(\omega)| d \sigma(\theta) d \sigma(\omega) \\
& \leq\left(\iint_{\Sigma \times \Sigma}\left|\left\langle P^{*} B_{\beta^{k} 2^{j}} \xi, \theta-\omega\right\rangle\right|^{-\epsilon q^{\prime}} d \sigma(\theta) d \sigma(\omega)\right)^{1 / q^{\prime}} \\
& \quad \times\|\Omega\|_{q}^{2} \leq C\left|B_{\beta^{k} 2^{j}} \xi\right|^{-\epsilon}\|\Omega\|_{q}^{2},
\end{aligned}
$$

where the last inequality follows from condition (3) of Section 1 (see [5, p. 553]). Therefore

$$
\int_{\beta^{k}}^{\beta^{k+1}}\left|\tau\left(B_{r} \xi\right)\right|^{2} d r / r \leq C\|\Omega\|_{q}^{2} \sum_{j=0}^{v}\left|B_{\beta^{k} 2^{j}} \xi\right|^{-\epsilon}
$$

(for $\left.0<\epsilon<\min \left(1 /\left(2 q^{\prime}\right), 1 / L\right)\right)$. If $s\left(B_{\beta^{k}} \xi\right) \geq 1, \quad\left|B_{\beta^{k} 2^{j}} \xi\right| \geq C\left(\beta^{k} 2^{j} s(\xi)\right)^{1 / a_{2}}$ $(0 \leq j \leq v)$. Thus

$$
\sum_{j=0}^{v}\left|B_{\beta^{k} 2^{j}} \xi\right|^{-\epsilon} \leq \sum_{j=0}^{v} C\left(\beta^{k} 2^{j} s(\xi)\right)^{-\epsilon / a_{2}} \leq C(\log \beta)\left(\beta^{k} s(\xi)\right)^{-\epsilon / a_{2}},
$$

where $C$ is independent of $q$. By (2.9) and (2.10) we have the estimate of Lemma 2.1 when $s\left(B_{\beta^{k}} \xi\right) \geq 1$. If $s\left(B_{\beta^{k}} \xi\right)<1$, the estimate of Lemma 2.1 follows from the inequality $|\tau(\xi)| \leq\|\Omega\|_{1}$. This completes the proof of Lemma 2.1. 
Now, by Hölder's inequality,

$$
\begin{aligned}
\left|\hat{\sigma}_{k}(\xi, \eta)\right| & =\left|\int_{\beta^{k}}^{\beta^{k+1}} \exp (-2 \pi i\langle\Gamma(r), \eta\rangle) h(r) \tau\left(B_{r} \xi\right) d r / r\right| \\
& \leq\left(\int_{\beta^{k}}^{\beta^{k+1}}|h(r)|^{s} d r / r\right)^{1 / s}\left(\int_{\beta^{k}}^{\beta^{k+1}}\left|\tau\left(B_{r} \xi\right)\right|^{s^{\prime}} d r / r\right)^{1 / s^{\prime}} \\
& \leq C(\log \beta)^{1 / s}\|h\|_{\Delta_{s}}\|\Omega\|_{1}^{\left(s^{\prime}-2\right) / s^{\prime}}\left(\int_{\beta^{k}}^{\beta^{k+1}}\left|\tau\left(B_{r} \xi\right)\right|^{2} d r / r\right)^{1 / s^{\prime}}
\end{aligned}
$$

where we have used the estimate $|\tau(\xi)| \leq\|\Omega\|_{1}$ to get the last inequality. By (2.11) and Lemma 2.1 we obtain (2.3). The estimate (2.4) can be proved similarly.

Let $B_{q s}=\left(1-\beta^{-\theta \epsilon_{0} / q^{\prime} s^{\prime}}\right)^{-1}$, where $\beta \geq 2, \theta \in(0,1)$ and $\epsilon_{0}$ is as in (2.3) and (2.4). To prove Theorems 1.1 and 1.3, we use the following result.

Proposition 2.3. Suppose that $\Omega \in L^{q}, q \in(1,2]$ and $h \in \Delta_{s}, s \in(1,2]$. Let $|1 / p-1 / 2|<(1-\theta) /\left(s^{\prime}(1+\theta)\right)$. Then

$$
\|T f\|_{p} \leq C(\log \beta)\|h\|_{\Delta_{s}}\|\Omega\|_{q} B_{q s} B_{q 2}^{\left|1 / p-1 / p^{\prime}\right|}\|f\|_{p},
$$

where $C$ is a constant independent of $\Omega, h, q, s$ and $\beta$.

Proposition 2.4. Suppose that $\Gamma \equiv 0$. Let $\Omega \in L^{q}, h \in \Delta_{s}, q, s \in(1,2]$. Then, for $p \in(1+\theta,(1+\theta) / \theta)$,

$$
\|T f\|_{p} \leq C(\log \beta)\|\Omega\|_{q}\|h\|_{\Delta_{s}} B_{q s}^{1+\left|1 / p-1 / p^{\prime}\right|}\|f\|_{p},
$$

where $C$ is a constant independent of $\Omega, h, q, s$ and $\beta$.

To prove Propositions 2.3 and 2.4, we need the following result.

Proposition 2.5. Let $\mu^{*}(f)(x)=\sup _{k}\left|\mu_{k} * f(x)\right|$. Let $\Omega \in L^{q}, q \in(1,2]$.

(1) If $h \in \Delta_{\infty}$, then for $p>1+\theta$,

$$
\left\|\mu^{*}(f)\right\|_{p} \leq C(\log \beta)\|\Omega\|_{q}\|h\|_{\Delta_{\infty}} B_{q 2}^{2 / p}\|f\|_{p},
$$

where $C$ is a constant independent of $\Omega, h, q$ and $\beta$.

(2) Suppose that $\Gamma \equiv 0$. Let $h \in \Delta_{s}, s \in(1,2]$. Then

$$
\left\|\mu^{*}(f)\right\|_{p} \leq C(\log \beta)\|\Omega\|_{q}\|h\|_{\Delta_{s}} B_{q s}^{2 / p}\|f\|_{p}
$$

for $p>1+\theta$, where $C$ is independent of $\Omega, q, h, s$ and $\beta$.

PROOF. Since the estimate $\left\|\mu^{*}(f)\right\|_{\infty} \leq C(\log \beta)\|\Omega\|_{1}\|h\|_{\Delta_{1}}\|f\|_{\infty}$ follows from (2.1), by interpolation, to prove (1) and (2) of Proposition 2.5 we may assume that $p \in(1+\theta, 2]$. 
First, we give a proof of part (1). Define measures $v_{k}$ on $\mathbb{R}^{n} \times \mathbb{R}^{m}$ by

$$
\hat{v}_{k}(\xi, \eta)=\hat{\mu}_{k}(\xi, \eta)-\hat{\Psi}_{k}(\xi, \eta),
$$

where $\hat{\Psi}_{k}(\xi, \eta)=\hat{\varphi}_{k}(\xi) \hat{\mu}_{k}(0, \eta)$ with $\varphi_{k}(x)=\beta^{-k \gamma} \varphi\left(A_{\beta^{-k}} x\right), \varphi \in C_{0}^{\infty}$. We assume that $\varphi$ is supported in $\{r(x) \leq 1\}, \hat{\varphi}(0)=1$ and $\varphi \geq 0$. Then by (2.1), (2.4) and (2.5), for $q, s \in(1,2]$,

$$
\left|\hat{v}_{k}(\xi, \eta)\right| \leq C(\log \beta)\|\Omega\|_{q}\|h\|_{\Delta_{s}} \min \left(1,\left(\beta^{k+d} s(\xi)\right)^{1 / b_{1}},\left(\beta^{k} s(\xi)\right)^{-\epsilon_{0} / q^{\prime} s^{\prime}}\right) .
$$

We may assume that $\epsilon_{0}$ is small enough so that $\epsilon_{0} / 4 \leq 1 / b_{1}$. Then

$$
\left|\hat{v}_{k}(\xi, \eta)\right| \leq C A \min \left(1,\left(\beta^{k+d} s(\xi)\right)^{\alpha},\left(\beta^{k} s(\xi)\right)^{-\alpha}\right),
$$

where $A=(\log \beta)\|\Omega\|_{q}\|h\|_{\Delta_{\infty}}$ and $\alpha=\epsilon_{0} /\left(2 q^{\prime}\right)$.

Let

$$
g(f)(x, z)=\left(\sum_{k=-\infty}^{\infty}\left|v_{k} * f(x, z)\right|^{2}\right)^{1 / 2} .
$$

Then $\mu^{*}(f) \leq g(f)+\Psi^{*}(|f|)$, where $\Psi^{*}(f)=\sup _{k}|| \Psi_{k}|* f|$. Let

$$
M g(x)=\sup _{t>0} t^{-\gamma} \int_{r(x-y)<t}|g(y)| d y
$$

be the Hardy-Littlewood maximal function on $\mathbb{R}^{n}$ with respect to the function $r$. By the $L^{p}$ boundedness of $M_{\Gamma}$ and $M$, it is easy to see that $\left\|\Psi^{*}(f)\right\|_{p} \leq C A\|f\|_{p}$ for $p>1$. Thus to prove Proposition 2.5(1) it suffices to show that

$$
\|g(f)\|_{p} \leq C A B^{2 / p}\|f\|_{p} \quad(p \in(1+\theta, 2]),
$$

where $A$ is as above and $B=B_{q 2}$. By a well-known property of Rademacher's functions, (2.13) follows from

$$
\left\|U_{\epsilon}(f)\right\|_{p} \leq C A B^{2 / p}\|f\|_{p} \quad(p \in(1+\theta, 2]),
$$

where $U_{\epsilon}(f)(x, z)=\sum \epsilon_{k} v_{k} * f(x, z)$ with $\epsilon=\left\{\epsilon_{k}\right\}, \epsilon_{k}=1$ or -1 (the inequality is uniform in $\epsilon$ ).

We define two sequences $\left\{r_{m}\right\}_{1}^{\infty}$ and $\left\{p_{m}\right\}_{1}^{\infty}$ by $p_{1}=2$ and

$$
\frac{1}{r_{m}}-\frac{1}{2}=\frac{1}{2 p_{m}}, \quad \frac{1}{p_{m+1}}=\frac{\theta}{2}+\frac{1-\theta}{r_{m}} \text { for } m \geq 1 .
$$

Then

$$
\frac{1}{p_{m+1}}=\frac{1}{2}+\frac{1-\theta}{2 p_{m}} \quad \text { for } m \geq 1 .
$$

Thus $1 / p_{m}=\left(1-\eta^{m}\right) /(1+\theta)$, where $\eta=(1-\theta) / 2$, so $\left\{p_{m}\right\}$ is decreasing and converges to $1+\theta$. 
For $j \geq 1$ we prove that

$$
\left\|U_{\epsilon}(f)\right\|_{p_{j}} \leq C_{j} A B^{2 / p_{j}}\|f\|_{p_{j}},
$$

using the Littlewood-Paley theory. Let $\left\{\psi_{k}\right\}_{-\infty}^{\infty}$ be a sequence of nonnegative functions in $C^{\infty}((0, \infty))$ such that

$$
\begin{gathered}
\operatorname{supp}\left(\psi_{k}\right) \subset\left[\beta^{-k-1}, \beta^{-k+1}\right], \quad \sum_{k} \psi_{k}(t)^{2}=1, \\
\left|(d / d t)^{j} \psi_{k}(t)\right| \leq c_{j} / t^{j},
\end{gathered}
$$

for $j=1,2, \ldots$, where $c_{j}$ is independent of $\beta \geq 2$. Define $S_{k}$ by

$$
\left(S_{k}(f) \hat{)}(\xi, \eta)=\psi_{k}(s(\xi)) \hat{f}(\xi, \eta) .\right.
$$

We write $U_{\epsilon}(f)=\sum_{j=-\infty}^{\infty} U_{j}(f)$, where $U_{j}(f)=\sum_{k=-\infty}^{\infty} \epsilon_{k} S_{j+k}\left(v_{k} * S_{j+k}(f)\right)$. Then by Plancherel's theorem and (2.12),

$$
\begin{aligned}
\left\|U_{j}(f)\right\|_{2}^{2} & \leq \sum_{k} C \iint_{D(j+k) \times \mathbb{R}^{m}}\left|\hat{v}_{k}(\xi, \eta)\right|^{2}|\hat{f}(\xi, \eta)|^{2} d \xi d \eta \\
& \leq C A^{2} \min \left(1, \beta^{-2(|j|-1-d) \alpha}\right) \sum_{k} \iint_{D(j+k) \times \mathbb{R}^{m}}|\hat{f}(\xi, \eta)|^{2} d \xi d \eta \\
& \leq C A^{2} \min \left(1, \beta^{-2(|j|-1-d) \alpha}\right)\|f\|_{2}^{2}
\end{aligned}
$$

where $D(k)=\left\{\xi \in \mathbb{R}^{n} \mid \beta^{-k-1}<s(\xi) \leq \beta^{-k+1}\right\}$. By (2.16),

$$
\begin{aligned}
\left\|U_{\epsilon}(f)\right\|_{2} & \leq \sum_{-\infty}^{\infty}\left\|U_{j}(f)\right\|_{2} \leq C \sum_{-\infty}^{\infty} A \min \left(1, \beta^{-(|j|-1-d) \alpha}\right)\|f\|_{2} \\
& \leq C A\left(1-\beta^{-\alpha}\right)^{-1}\|f\|_{2} .
\end{aligned}
$$

If we denote by $A(m)$ the estimate of (2.15) for $j=m$, this proves $A(1)$.

Now we assume $A(m)$ and derive $A(m+1)$ from $A(m)$. Note that

$$
\nu^{*}(f) \leq \mu^{*}(|f|)+\Psi^{*}(|f|) \leq g(|f|)(x)+2 \Psi^{*}(|f|),
$$

where $v^{*}(f)(x)=\sup _{k} \| v_{k}|* f(x)|$. Since $\|g(f)\|_{p_{m}} \leq C A B^{2 / p_{m}}\|f\|_{p_{m}}$ by $A(m)$,

$$
\left\|v^{*}(f)\right\|_{p_{m}} \leq C A B^{2 / p_{m}}\|f\|_{p_{m}} .
$$

Also, $\left\|v_{k}\right\| \leq C A$ by (2.1). Thus, by the proof of Lemma for [5, Theorem B, p. 544], the following vector-valued inequality holds:

$$
\begin{aligned}
\left\|\left(\sum\left|v_{k} * g_{k}\right|^{2}\right)^{1 / 2}\right\|_{r_{m}} & \leq C\left(A B^{2 / p_{m}} \sup _{k}\left\|v_{k}\right\|\right)^{1 / 2}\left\|\left(\sum\left|g_{k}\right|^{2}\right)^{1 / 2}\right\|_{r_{m}} \\
& \leq C A B^{1 / p_{m}}\left\|\left(\sum\left|g_{k}\right|^{2}\right)^{1 / 2}\right\|_{r_{m}} .
\end{aligned}
$$


By (2.18) and the Littlewood-Paley inequality,

$$
\begin{aligned}
\left\|U_{j}(f)\right\|_{r_{m}} & \leq C\left\|\left(\sum_{k}\left|v_{k} * S_{j+k}(f)\right|^{2}\right)^{1 / 2}\right\|_{r_{m}} \\
& \leq C A B^{1 / p_{m}}\|f\|_{r_{m}} .
\end{aligned}
$$

Here we note that the bounds for the Littlewood-Paley inequality are independent of $\beta \geq 2$. Interpolating between (2.16) and (2.19),

$$
\left\|U_{j}(f)\right\|_{p_{m+1}} \leq C A B^{(1-\theta) / p_{m}} \min \left(1, \beta^{-\theta \alpha(|j|-1-d)}\right)\|f\|_{p_{m+1}} .
$$

Thus

$$
\begin{aligned}
\left\|U_{\epsilon}(f)\right\|_{p_{m+1}} & \leq \sum_{j}\left\|U_{j}(f)\right\|_{p_{m+1}} \leq C A B^{(1-\theta) / p_{m}}\left(1-\beta^{-\theta \alpha}\right)^{-1}\|f\|_{p_{m+1}} \\
& \leq C A B^{2 / p_{m+1}}\|f\|_{p_{m+1}},
\end{aligned}
$$

which proves $A(m+1)$. By induction, this completes the proof of (2.15).

We now prove (2.14). Let $p \in(1+\theta, 2]$ and let $\left\{p_{m}\right\}_{1}^{\infty}$ be as in (2.15). Then we have $p_{N+1}<p \leq p_{N}$ for some $N$. By interpolation between the estimates in (2.15) for $j=N$ and $j=N+1,(2.14)$ holds. This completes the proof of part (1) of Proposition 2.5.

Part (2) of Proposition 2.5 can be proved in the same way. We take $A=(\log \beta)\|\Omega\|_{q}\|h\|_{\Delta_{s}}$ and $\alpha=\epsilon_{0} / q^{\prime} s^{\prime}$ in (2.12). Then, since

$$
\left\|\Psi^{*}(f)\right\|_{p} \leq C(\log \beta)\|\Omega\|_{1}\|h\|_{\Delta_{1}}\|f\|_{p} \quad \text { for } p>1
$$

if $\Gamma \equiv 0$, the proof of part (1) can be used to get (2.13) with $A=(\log \beta)\|\Omega\|_{q}\|h\|_{\Delta_{s}}$ as above and $B=B_{q s}$, and the conclusion of part (2) follows from (2.13).

Proof of Proposition 2.3. To prove Proposition 2.3 we may assume that $1<s<$ 2. As in [1], here we apply an idea in the proof of [6, Theorem 7.5]. We consider measures $\tau_{k}$ defined by

$$
\begin{aligned}
\hat{\tau}_{k}(\xi, \eta)= & \int_{E_{k}} \exp (-2 \pi i\langle y, \xi\rangle) \\
& \times \exp (-2 \pi i\langle\Gamma(r(y)), \eta\rangle)|h(r(y))|^{2-s}\left|\Omega\left(y^{\prime}\right)\right| r(y)^{-\gamma} d y .
\end{aligned}
$$

Then the Schwarz inequality implies that

$$
\left|\sigma_{k} * f\right|^{2} \leq C(\log \beta)\|h\|_{\Delta_{s}}^{s}\|\Omega\|_{1} \tau_{k} *|f|^{2} .
$$

Define measures $\lambda_{k}$ by

$$
\hat{\lambda}_{k}(\xi, \eta)=\int_{E_{k}} \exp (-2 \pi i\langle y, \xi\rangle) \exp (-2 \pi i\langle\Gamma(r(y)), \eta\rangle)\left|\Omega\left(y^{\prime}\right)\right| r(y)^{-\gamma} d y .
$$


Since $|h|^{2-s} \in \Delta_{s /(2-s)}$ and $\left\||h|^{2-s}\right\|_{\Delta_{s /(2-s)}}=\|h\|_{\Delta_{s}}^{2-s}$, if $u=s /(2-s)$ then, by Hölder's inequality,

$$
\left|\tau_{k} * f\right| \leq C(\log \beta)^{1 / u}\|h\|_{\Delta_{s}}^{2-s}\|\Omega\|_{1}^{1 / u}\left(\lambda_{k} *|f|^{u^{\prime}}\right)^{1 / u^{\prime}} .
$$

Therefore, if $1+\theta<r / u^{\prime}=2 r(s-1) / s$, by applying (1) of Proposition 2.5 to $\left\{\lambda_{k}\right\}$ we see that

$$
\left\|\tau^{*}(f)\right\|_{r} \leq C(\log \beta)\|h\|_{\Delta_{s}}^{2-s}\|\Omega\|_{q} B_{q 2}^{2 / r}\|f\|_{r},
$$

where $\tau^{*}(f)=\sup _{k}\left|\tau_{k} * f\right|$. Thus, if $|1 / v-1 / 2|=1 / 2 r<1 /\left(s^{\prime}(1+\theta)\right)$, using (2.20), (2.21) and arguing as in the proof of Lemma for [5, Theorem B, p. 544], we see that

$$
\left\|\left(\sum\left|\sigma_{k} * g_{k}\right|^{2}\right)^{1 / 2}\right\|_{v} \leq C(\log \beta)\|h\|_{\Delta_{s}}\|\Omega\|_{q} B_{q 2}^{1 / r}\left\|\left(\sum\left|g_{k}\right|^{2}\right)^{1 / 2}\right\|_{v} .
$$

We decompose $T f=\sum_{j=-\infty}^{\infty} V_{j} f$, where $V_{j} f=\sum_{k=-\infty}^{\infty} S_{j+k}\left(\sigma_{k} * S_{j+k}(f)\right)$. Then, using (2.22) and the Littlewood-Paley theory,

$$
\left\|V_{j} f\right\|_{v} \leq C(\log \beta)\|h\|_{\Delta_{s}}\|\Omega\|_{q} B_{q 2}^{1 / r}\|f\|_{v},
$$

where $|1 / v-1 / 2|=1 / 2 r<1 /\left(s^{\prime}(1+\theta)\right)$. On the other hand, by (2.1)-(2.3),

$$
\left|\hat{\sigma}_{k}(\xi, \eta)\right| \leq C(\log \beta)\|\Omega\|_{q}\|h\|_{\Delta_{s}} \min \left(1,\left(\beta^{k+d} s(\xi)\right)^{\kappa},\left(\beta^{k} s(\xi)\right)^{-\kappa}\right),
$$

where $\kappa=\epsilon_{0} / q^{\prime} s^{\prime}$, and hence, much as in the proof of (2.16), we can show that

$$
\left\|V_{j} f\right\|_{2} \leq C(\log \beta)\|h\|_{\Delta_{s}}\|\Omega\|_{q} \min \left(1, \beta^{-(|j|-1-d) \kappa}\right)\|f\|_{2} .
$$

If $|1 / p-1 / 2|<(1-\theta) /\left(s^{\prime}(1+\theta)\right)$, then we can find numbers $v$ and $r$ such that $|1 / v-1 / 2|=1 / 2 r<1 /\left(s^{\prime}(1+\theta)\right)$ and $1 / p=\theta / 2+(1-\theta) / v$. Thus, interpolating between (2.23) and (2.24),

$$
\left\|V_{j} f\right\|_{p} \leq C(\log \beta)\|h\|_{\Delta_{s}}\|\Omega\|_{q} B_{q 2}^{(1-\theta) / r} \min \left(1, \beta^{-\theta(|j|-1-d) \kappa}\right)\|f\|_{p} .
$$

Therefore

$$
\|T f\|_{p} \leq \sum_{j}\left\|V_{j} f\right\|_{p} \leq C(\log \beta)\|h\|_{\Delta_{s}}\|\Omega\|_{q} B_{q 2}^{(1-\theta) / r} B_{q s}\|f\|_{p} .
$$

This completes the proof of Proposition 2.3, since $(1-\theta) / r=\left|1 / p-1 / p^{\prime}\right|$.

Proof of Proposition 2.4. The $L^{2}$ estimates follow from Proposition 2.3 , so on account of duality and interpolation we may assume that $1+\theta<p \leq 4 /(3-\theta)$. For $p_{0} \in(1+\theta, 4 /(3-\theta)]$ we can find $r \in(1+\theta, 2]$ such that $1 / p_{0}=1 / 2+$ $(1-\theta) / 2 r$. If $\Gamma \equiv 0$, by (2) of Proposition 2.5 and (2.1), arguing as in (2.18), we obtain (2.22) with $B_{q 2}$ replaced by $B_{q s}$ for the number $v$ satisfying $1 / v-1 / 2=1 / 2 r$ (note that $1 / p_{0}=\theta / 2+(1-\theta) / v$ ). Thus, arguing as in the proof of Proposition 2.3, we obtain (2.25) with $p=p_{0}$ and $B_{q s}$ in place of $B_{q 2}$. This ends the proof of Proposition 2.4. 
Now we can give proofs of Theorems 1.1 and 1.3. To prove Theorem 1.1, we may assume that $1<s \leq 2$. Let $\beta=2^{q^{\prime}}$ in Proposition 2.3. Then, since $\theta$ is an arbitrary number in $(0,1)$, we have Theorem 1.1 for $s \in(1,2]$.

Next, take $\beta=2^{q^{\prime} s^{\prime}}$ in Proposition 2.4. Then

$$
\|T f\|_{p} \leq C(q-1)^{-1}(s-1)^{-1}\|\Omega\|_{q}\|h\|_{\Delta_{s}}\|f\|_{p}
$$

for $p \in(1, \infty)$, since $(1+\theta,(1+\theta) / \theta) \rightarrow(1, \infty)$ as $\theta \rightarrow 0$. From this the result for $S$ in Theorem 1.3 follows if we take functions of the form $f(x, z)=k(x) g(z)$.

\section{Extrapolation}

We can prove Theorems 1.2 and 1.4 by an extrapolation method similar to that used in [14]. We give a proof of Theorem 1.4 for the sake of completeness (Theorem 1.2 can be proved in the same way). We fix $p \in(1, \infty)$ and $f$ with $\|f\|_{p} \leq 1$. Let $S$ be as in (1.2). We also write $S f=S_{h, \Omega}(f)$. Put $U(h, \Omega)=\left\|S_{h, \Omega}(f)\right\|_{p}$. Then we see that

$$
\begin{aligned}
U\left(h, \Omega_{1}+\Omega_{2}\right) & \leq U\left(h, \Omega_{1}\right)+U\left(h, \Omega_{1}\right), \\
U\left(h_{1}+h_{2}, \Omega\right) & \leq U\left(h_{1}, \Omega\right)+U\left(h_{2}, \Omega\right),
\end{aligned}
$$

for appropriate functions $\Omega, h, \Omega_{1}, \Omega_{2}, h_{1}$ and $h_{2}$. Set

$$
\begin{aligned}
E_{1} & =\left\{r \in \mathbb{R}_{+}|| h(r) \mid \leq 2\right\}, \\
E_{m} & =\left\{r \in \mathbb{R}_{+}\left|2^{m-1}<\right| h(r) \mid \leq 2^{m}\right\} \quad \text { for } m \geq 2 .
\end{aligned}
$$

Then $h=\sum_{m=1}^{\infty} h \chi_{E_{m}}$. Put $e_{m}=\sigma\left(F_{m}\right)$ for $m \geq 1$, where

$$
\begin{aligned}
F_{m} & =\left\{\theta \in \Sigma\left|2^{m-1}<\right| \Omega(\theta) \mid \leq 2^{m}\right\} \quad \text { for } m \geq 2, \\
F_{1} & =\{\theta \in \Sigma|| \Omega(\theta) \mid \leq 2\} .
\end{aligned}
$$

Let $\Omega_{m}=\Omega \chi_{F_{m}}-\sigma(\Sigma)^{-1} \int_{F_{m}} \Omega d \sigma$. Then $\Omega=\sum_{m=1}^{\infty} \Omega_{m}$. Note that $\int_{\Sigma} \Omega_{m} d \sigma=$ 0 . Applying Theorem 1.3, we see that

$$
U\left(h \chi_{E_{m}}, \Omega_{j}\right) \leq C(q-1)^{-1}(s-1)^{-1}\left\|h \chi_{E_{m}}\right\|_{\Delta_{s}}\left\|\Omega_{j}\right\|_{q}
$$

for all $s, q \in(1,2]$.

Now we follow the extrapolation argument of Zygmund [18, Ch. XII, pp. 119-120]. For $k \in \mathbb{Z}$, put

$$
\begin{aligned}
E(k, m) & =\left\{r \in\left(2^{k}, 2^{k+1}\right]\left|2^{m-1}<\right| h(r) \mid \leq 2^{m}\right\} \quad \text { for } m \geq 2, \\
E(k, 1) & =\left\{r \in\left(2^{k}, 2^{k+1}\right]|0<| h(r) \mid \leq 2\right\} .
\end{aligned}
$$

Then

$$
\begin{aligned}
\int_{E(k, m)}|h(r)|^{(m+1) / m} d r / r & \leq C m^{-a} \int_{E(k, m)}|h(r)|(\log (2+|h(r)|))^{a} d r / r \\
& \leq C m^{-a} L_{a}(h),
\end{aligned}
$$


and hence

$$
\left\|h \chi_{E_{m}}\right\|_{\Delta_{1+1 / m}} \leq C m^{-a m /(m+1)} L_{a}(h)^{m /(m+1)}
$$

for $m \geq 1$. Also

$$
\left\|\Omega_{j}\right\|_{1+1 / j} \leq C 2^{j} e_{j}^{j /(j+1)} .
$$

From (3.1)-(3.4),

$$
\begin{aligned}
U(h, \Omega) & \leq \sum_{m \geq 1} \sum_{j \geq 1} U\left(h \chi_{E_{m}}, \Omega_{j}\right) \leq C \sum_{m \geq 1} \sum_{j \geq 1} j m\left\|h \chi_{E_{m}}\right\|_{\Delta_{1+1 / m}}\left\|\Omega_{j}\right\|_{1+1 / j} \\
& \leq C\left(1+L_{a}(h)\right) \sum_{m \geq 1} \sum_{j \geq 1} m^{1-a m /(m+1)} j 2^{j} e_{j}^{j /(j+1)} \\
& =C\left(1+L_{a}(h)\right)\left(\sum_{m \geq 1} m^{1-a m /(m+1)}\right)\left(\sum_{j \geq 1} j 2^{j} e_{j}^{j /(j+1)}\right) .
\end{aligned}
$$

When $a>2$, it is easy to see that $\sum_{m \geq 1} m^{1-a m /(m+1)}<\infty$. Also,

$$
\begin{aligned}
\sum_{j \geq 1} j 2^{j} e_{j}^{j /(j+1)} & =\sum_{e_{j}<3^{-j}} j 2^{j} e_{j}^{j /(j+1)}+\sum_{e_{j} \geq 3^{-j}} j 2^{j} e_{j}^{j /(j+1)} \\
& \leq \sum_{j \geq 1} j 2^{j} 3^{-j^{2} /(j+1)}+\sum_{j \geq 1} j 2^{j} e_{j} 3^{j /(j+1)} \\
& \leq C+C \int_{\Sigma}|\Omega(\theta)| \log (2+|\Omega(\theta)|) d \sigma(\theta) .
\end{aligned}
$$

Collecting the results, we conclude the proof of Theorem 1.4.

REMARK. For a positive number $a$ and a function $h$ on $\mathbb{R}_{+}$, let

$$
N_{a}(h)=\sum_{m \geq 1} m^{a} 2^{m} d_{m}(h),
$$

where $d_{m}(h)=\sup _{k \in \mathbb{Z}} 2^{-k}|E(k, m)|\left(E(k, m)\right.$ is as above). We define a class $\mathcal{N}_{a}$ to be the space of all measurable functions $h$ on $\mathbb{R}_{+}$which satisfy $N_{a}(h)<\infty$. Then it can be shown that if $h \in \mathcal{L}_{a}$ for some $a>2$, then $h \in \mathcal{N}_{1}$. By a method similar to that used in this section, we can show the $L^{p}$ boundedness of $S$ in Theorem 1.4 under a less restrictive condition that $h \in \mathcal{N}_{1}$ and $\Omega \in L \log L$ (see [14]).

\section{An estimate for a trigonometric integral}

Let $A$ be an $n \times n$ real matrix and

$$
\phi_{A}(t)=\left(t-\gamma_{1}\right)^{m_{1}}\left(t-\gamma_{2}\right)^{m_{2}} \cdots\left(t-\gamma_{k}\right)^{m_{k}}
$$

be the minimal polynomial of $A$, where $\gamma_{i} \neq \gamma_{j}$ if $i \neq j$. Let $a_{i}(t)=\left(t-\gamma_{i}\right)^{m_{i}}$ for $i=1,2, \ldots, k$. Then we can find polynomials $b_{i}(t)(i=1,2, \ldots, k)$ such that

$$
\frac{1}{\phi_{A}(t)}=\sum_{i=1}^{k} \frac{b_{i}(t)}{a_{i}(t)} \text {. }
$$


For each $i, 1 \leq i \leq k$, let $P_{i}$ be the polynomial defined by

$$
P_{i}(t)=\frac{b_{i}(t)}{a_{i}(t)} \phi_{A}(t) .
$$

We consider the $n \times n$ matrices $P_{i}(A)$, which are defined as usual (see [8]).

Let

$$
V_{i}=\left\{z \in \mathbb{C}^{n} \mid\left(A-\gamma_{i} E\right)^{m_{i}} z=0\right\} \quad(i=1,2, \ldots, k),
$$

where $E$ denotes the unit matrix. Then the vector space $\mathbb{C}^{n}$ can be decomposed into a direct sum as

$$
\mathbb{C}^{n}=V_{1} \oplus V_{2} \oplus \cdots \oplus V_{k}
$$

Each of the matrices $P_{i}(A)$ is the projection onto $V_{i}$; indeed, $P_{i}(A) z \in V_{i}$ for all $z \in \mathbb{C}^{n}$, for $i=1,2, \ldots, k$, and

$$
\begin{gathered}
P_{1}(A)+P_{2}(A)+\cdots+P_{k}(A)=E \\
P_{i}^{2}(A)=P_{i}(A), \quad P_{i}(A) P_{j}(A)=0 \quad \text { if } i \neq j \quad(1 \leq i, j \leq k) .
\end{gathered}
$$

For $z=\left(z_{i}\right)$ and $w=\left(w_{i}\right)$ in $\mathbb{C}^{n}$, we write $\langle z, w\rangle=\sum_{i=1}^{n} z_{i} w_{i}$. Let

$$
J(A, \eta, \zeta)=\sum_{i=1}^{k} \sum_{j=0}^{m_{i}-1}\left|\left\langle\left(A-\gamma_{i} E\right)^{j} P_{i}(A) \eta, A^{*} \zeta\right\rangle\right|
$$

for $\eta, \zeta \in \mathbb{R}^{n}$. In this section, we prove the following result.

Theorem 4.1. Let $\eta, \zeta \in \mathbb{R}^{n} \backslash\{0\}$ and $0<a<b$. Suppose that $J(A, \eta, \zeta) \neq 0$ and the numbers $a, b$ are in a fixed compact subinterval of $(0, \infty)$. Then

$$
\left|\int_{a}^{b} \exp \left(i\left\langle t^{A} \eta, \zeta\right\rangle\right) d t\right| \leq C J(A, \eta, \zeta)^{-1 / N},
$$

where $N=\operatorname{deg} \phi_{A}=m_{1}+m_{2}+\cdots+m_{k}$ and the constant $C$ is independent of $\eta, \zeta$, $a$ and $b$.

Since $\sum_{i=1}^{k} P_{i}(A)=E$, using the triangle inequality,

$$
\left|\left\langle\eta, A^{*} \zeta\right\rangle\right| \leq \sum_{i=1}^{k}\left|\left\langle P_{i}(A) \eta, A^{*} \zeta\right\rangle\right| \leq J(A, \eta, \zeta) .
$$

Therefore Theorem 4.1 implies the following result.

Corollary 4.2. Let $\eta, \zeta, a, b$ and $N$ be as in Theorem 4.1. Then

$$
\left|\int_{a}^{b} \exp \left(i\left\langle t^{A} \eta, \zeta\right\rangle\right) d t\right| \leq C|\langle A \eta, \zeta\rangle|^{-1 / N}
$$

when $\langle A \eta, \zeta\rangle \neq 0$.

This is used to prove Lemma 2.2 in Section 2. 
We define the curve $X(t)=t^{A} \eta$ for a fixed $\eta \in \mathbb{R}^{n} \backslash\{0\}$. Then Stein and Wainger [17] proved the following theorem (see [11, 16] for related results).

THEOREM A. Suppose that the curve $X$ does not lie in an affine hyperplane. Then

$$
\left|\int_{a}^{b} \exp (i\langle X(t), \zeta\rangle) d t\right| \leq C|\zeta|^{-1 / n},
$$

where $C$ is independent of $\zeta \in \mathbb{R}^{n} \backslash\{0\}$; furthermore, if a and $b$ are in a fixed compact subinterval of $(0, \infty)$, the constant $C$ is also independent of $a$ and $b$.

Evidently Theorem 4.1 implies Theorem A. Since $P_{i}(A) z \in V_{i}$ for all $z \in \mathbb{C}^{n}$,

$$
\left(A-\gamma_{i} E\right)^{m} P_{i}(A)=0 \quad \text { if } m \geq m_{i}(i=1,2, \ldots, k) .
$$

Therefore

$$
\begin{aligned}
\exp ((\log t) A) P_{i}(A) & =\exp \left((\log t) \gamma_{i} E\right) \exp \left((\log t)\left(A-\gamma_{i} E\right)\right) P_{i}(A) \\
& =t^{\gamma_{i}} \sum_{j=0}^{m_{i}-1} \frac{(\log t)^{j}}{j !}\left(A-\gamma_{i} E\right)^{j} P_{i}(A)
\end{aligned}
$$

Thus, using $\sum_{i=1}^{k} P_{i}(A)=E$,

$$
t^{A}=\sum_{i=1}^{k} t^{\gamma_{i}}\left[\sum_{j=0}^{m_{i}-1} \frac{(\log t)^{j}}{j !}\left(A-\gamma_{i} E\right)^{j}\right] P_{i}(A) .
$$

The assumption on $X$ of Theorem A can be rephrased as follows: the function $\psi(t)=\left\langle t^{A} \eta, \zeta\right\rangle$ is not a constant function on $(0, \infty)$ for every $\zeta \in \mathbb{R}^{n} \backslash\{0\}$. If $\psi(t)$ is not a constant function, then $\psi^{\prime}(t)$ is not identically 0 . Thus, since $t(d / d t) \psi(t)=$ $\left\langle t^{A} \eta, A^{*} \zeta\right\rangle$, by (4.2) we have $J(A, \eta, \zeta)>0$, where $J(A, \eta, \zeta)$ is as in (4.1). Let $C_{0}=\min _{|\zeta|=1} J(A, \eta, \zeta)$ and note that $C_{0}>0$. Then, from Theorem 4.1, it follows that

$$
\left|\int_{a}^{b} \exp (i\langle X(t), \zeta\rangle) d t\right| \leq C C_{0}^{-1 / N}|\zeta|^{-1 / N} .
$$

This implies Theorem A, since $N \leq n$ (in fact, it is not difficult to see that $N=n$ if $X$ satisfies the assumption of Theorem A).

We conclude this paper with a proof of Theorem 4.1. Let $I=[\alpha, \beta]$ be a compact interval in $\mathbb{R}$. Consider the differential equation

$$
y^{(k)}+a_{1} y^{(k-1)}+a_{2} y^{(k-2)}+\cdots+a_{k} y=0 \text { on } I,
$$

where $a_{1}, a_{2}, \ldots, a_{k}$ are complex constants. Let $\left\{\varphi_{1}, \varphi_{2}, \ldots, \varphi_{k}\right\}$ be a basis for the space $S$ of all solutions of (4.3). Then in order to prove Theorem 4.1 we require the following result. 
Proposition 4.3. Let $\varphi$ be a real-valued function such that $\varphi^{\prime} \in S$. Suppose that $\varphi^{\prime}=d_{1} \varphi_{1}+d_{2} \varphi_{2}+\cdots+d_{k} \varphi_{k}$, where $d_{1}, d_{2}, \ldots, d_{k}$ are complex constants, which are uniquely determined by $\varphi^{\prime}$. Then

$$
\left|\int_{\alpha}^{\beta} e^{i \varphi(t)} d t\right| \leq C\left(\left|d_{1}\right|+\left|d_{2}\right|+\cdots+\left|d_{k}\right|\right)^{-1 / k},
$$

where $C$ is independent of $\varphi$; also the constant $C$ is independent of $\alpha, \beta$ if they are within a fixed finite interval of $\mathbb{R}$.

To prove Proposition 4.3 we use the following two lemmas, both of which are well known.

LEMMA 4.4. Let $\varphi$ be a solution of (4.3). Suppose that $\varphi$ is not identically 0 . Then there exists a positive integer $K$ independent of $\varphi$ such that $\varphi$ has at most $K$ zeros in $I$.

Lemma 4.5 (van der Corput). Let $f:[c, d] \rightarrow \mathbb{R}$ and $f \in C^{j}([c, d])$ for some positive integer $j$, where $[c, d]$ is an arbitrary compact interval in $\mathbb{R}$. Suppose that $\inf _{u \in[c, d]}\left|(d / d u)^{j} f(u)\right| \geq \lambda>0$. When $j=1$, we further assume that $f^{\prime}$ is monotone on $[c, d]$. Then

$$
\left|\int_{c}^{d} e^{i f(u)} d u\right| \leq C_{j} \lambda^{-1 / j},
$$

where $C_{j}$ is a positive constant depending only on $j .($ See $[17,18]$.)

We now give a proof of Proposition 4.3. We consider linear combinations $c_{1} \varphi_{1}+c_{2} \varphi_{2}+\cdots+c_{k} \varphi_{k}$, where $c_{1}, c_{2}, \ldots, c_{k} \in \mathbb{C}$. We write $\psi=c_{1} \varphi_{1}+c_{2} \varphi_{2}+$ $\cdots+c_{k} \varphi_{k}$ and define

$$
\begin{aligned}
& N_{1}(\psi)=\left|c_{1}\right|+\left|c_{2}\right|+\cdots+\left|c_{k}\right| \\
& N_{2}(\psi)=\min _{t \in I}\left(|\psi(t)|+\left|\psi^{\prime}(t)\right|+\cdots+\left|\psi^{(k-1)}(t)\right|\right) .
\end{aligned}
$$

Let $U=\left\{\left(c_{1}, c_{2}, \ldots, c_{k}\right) \in \mathbb{C}^{k}|| c_{1}|+| c_{2}|+\cdots+| c_{k} \mid=1\right\} . \quad$ We consider a function $F$ on $I \times U$ defined by

$$
F\left(t, c_{1}, c_{2}, \ldots, c_{k}\right)=|\psi(t)|+\left|\psi^{\prime}(t)\right|+\cdots+\left|\psi^{(k-1)}(t)\right| .
$$

Then the function $F$ is continuous and positive on $I \times U$ (see [4]). Thus, if we put

$$
C_{0}=\min _{\left(t, c_{1}, c_{2}, \ldots, c_{k}\right) \in I \times U} F\left(t, c_{1}, c_{2}, \ldots, c_{k}\right),
$$

then $C_{0}>0$ and $N_{2}(\psi) \geq C_{0} N_{1}(\psi)$.

Therefore, if $\varphi$ is as in Proposition 4.3,

$$
\min _{t \in I}\left(\left|\varphi^{\prime}(t)\right|+\left|\varphi^{\prime \prime}(t)\right|+\cdots+\left|\varphi^{(k)}(t)\right|\right) \geq C_{0} N_{1}\left(\varphi^{\prime}\right) .
$$


By (4.4), for any $t \in I$, there exists $\ell \in\{1,2, \ldots, k\}$ such that

$$
\left|(d / d t)^{\ell} \varphi(t)\right| \geq C N_{1}\left(\varphi^{\prime}\right), \quad C>0 .
$$

By a suitable application of Lemma 4.4, we can decompose $I=\cup_{m=1}^{H} I_{m}$, where $H$ is a positive integer independent of $\varphi$ and $\left\{I_{m}\right\}$ is a family of nonoverlapping subintervals of $I$ such that for any interval $I_{m}$ there exists $\ell_{m} \in\{1,2, \ldots, k\}$ satisfying $\left|(d / d t)^{\ell_{m}} \varphi(t)\right| \geq\left|(d / d t)^{j} \varphi(t)\right|$ on $I_{m}$ for all $j \in\{1,2, \ldots, k\}$, so $\left|(d / d t)^{\ell_{m}} \varphi(t)\right| \geq$ $C N_{1}\left(\varphi^{\prime}\right)$ on $I_{m}$, and such that $\varphi^{\prime}$ is monotone on each $I_{m}$. Therefore, by Lemma 4.5,

$$
\begin{aligned}
\left|\int_{\alpha}^{\beta} e^{i \varphi(t)} d t\right| & =\left|\sum_{m=1}^{H} \int_{I_{m}} e^{i \varphi(t)} d t\right| \leq C \sum_{m=1}^{H} \min \left(\left|I_{m}\right|, N_{1}\left(\varphi^{\prime}\right)^{-1 / \ell_{m}}\right) \\
& \leq C N_{1}\left(\varphi^{\prime}\right)^{-1 / k} .
\end{aligned}
$$

Since $N_{1}\left(\varphi^{\prime}\right)=\left|d_{1}\right|+\left|d_{2}\right|+\cdots+\left|d_{k}\right|$, this completes the proof of Proposition 4.3.

PRoOF OF THEOREM 4.1. By the change of variables $t=e^{s}$ and an integration by parts argument, to prove Theorem 4.1 it suffices to show that

$$
\left|\int_{\alpha}^{\beta} \exp \left(i\left\langle e^{t A} \eta, \zeta\right\rangle\right) d t\right| \leq C J(A, \eta, \zeta)^{-1 / N}
$$

for an appropriate constant $C>0$, where $[\alpha, \beta]$ is an arbitrary compact interval in $\mathbb{R}$. Let $\psi(t)=\left\langle e^{t A} \eta, \zeta\right\rangle$. Then $\psi^{\prime}(t)=\left\langle e^{t A} \eta, A^{*} \zeta\right\rangle$, and hence, by (4.2),

$$
\psi^{\prime}(t)=\sum_{i=1}^{k} \sum_{j=0}^{m_{i}-1} c_{i j}(\eta, \zeta) t^{j} e^{\gamma_{i} t}
$$

where

$$
c_{i j}(\eta, \zeta)=\frac{1}{j !}\left\langle\left(A-\gamma_{i} E\right)^{j} P_{i}(A) \eta, A^{*} \zeta\right\rangle
$$

It is known that $N$ functions $t^{j} e^{\gamma_{i} t}\left(0 \leq j \leq m_{i}-1,1 \leq i \leq k\right)$ form a basis for the space of solutions for the ordinary differential equation of order $N$ with characteristic polynomial $\phi_{A}$ (see [4]). Thus, the estimate (4.5) immediately follows from Proposition 4.3, since $\sum_{i=1}^{k} \sum_{j=0}^{m_{i}-1}\left|c_{i j}(\eta, \zeta)\right| \approx J(A, \eta, \zeta)$.

\section{References}

[1] A. Al-Salman and Y. Pan, 'Singular integrals with rough kernels in $L \log L\left(S^{n-1}\right)$ ', J. London Math. Soc. (2) 66 (2002), 153-174.

[2] A. P. Calderón and A. Torchinsky, 'Parabolic maximal functions associated with a distribution', Adv. Math. 16 (1975), 1-64.

[3] A. P. Calderón and A. Zygmund, 'On singular integrals', Amer. J. Math. 78 (1956), 289-309.

[4] E. A. Coddington, An Introduction to Ordinary Differential Equations (Prentice-Hall, Englewood Cliffs, NJ, 1961). 
[5] J. Duoandikoetxea and J. L. Rubio de Francia, 'Maximal and singular integral operators via Fourier transform estimates', Invent. Math. 84 (1986), 541-561.

[6] D. Fan and Y. Pan, 'Singular integral operators with rough kernels supported by subvarieties', Amer. J. Math. 119 (1997), 799-839.

[7] R. Fefferman, 'A note on singular integrals', Proc. Amer. Math. Soc. 74 (1979), 266-270.

[8] S. Friedberg, A. Insel and L. Spence, Linear Algebra (Prentice-Hall, Englewood Cliffs, NJ, 1979).

[9] S. Hofmann, 'Weighted norm inequalities and vector valued inequalities for certain rough operators', Indiana Univ. Math. J. 42 (1993), 1-14.

[10] W. Kim, S. Wainger, J. Wright and S. Ziesler, 'Singular integrals and maximal functions associated to surfaces of revolution', Bull. London Math. Soc. 28 (1996), 291-296.

[11] A. Nagel and S. Wainger, ' $L^{2}$ boundedness of Hilbert transforms along surfaces and convolution operators homogeneous with respect to a multiple parameter group', Amer. J. Math. 99 (1977), 761-785.

[12] J. Namazi, 'On a singular integral', Proc. Amer. Math. Soc. 96 (1986), 421-424.

[13] N. Rivière, 'Singular integrals and multiplier operators', Ark. Mat. 9 (1971), 243-278.

[14] S. Sato, 'Estimates for singular integrals and extrapolation', Studia Math. 192 (2009), 219-233.

[15] E. M. Stein, Harmonic Analysis: Real-Variable Methods, Orthogonality and Oscillatory Integrals (Princeton University Press, Princeton, NJ, 1993).

[16] E. M. Stein and S. Wainger, 'The estimation of an integral arising in multiplier transformations', Studia Math. 35 (1970), 101-104.

[17] _ 'Problems in harmonic analysis related to curvature', Bull. Amer. Math. Soc. 84 (1978), $1239-1295$.

[18] A. Zygmund, Trigonometric Series, 2nd edn (Cambridge University Press, Cambridge, 1977).

\section{SHUICHI SATO, Department of Mathematics, Faculty of Education, Kanazawa University, Kanazawa 920-1192, Japan \\ e-mail: shuichi@kenroku.kanazawa-u.ac.jp}

